General Principles of Tumor Immunotherapy 


\title{
General Principles of Tumor Immunotherapy
}

\section{Basic and Clinical Applications of Tumor Immunology}

\author{
Edited by \\ Howard L. Kaufman \\ The Tumor Immunology Laboratory, \\ Division of Surgical Oncology, \\ Columbia University, \\ New York, NY \\ and \\ Jedd D. Wolchok \\ Department of Medicine, \\ Memorial Sloan-Kettering Cancer Center, \\ Weill Medical College of Cornell University, \\ New York, NY \\ 算 Springer
}


A C.I.P. Catalogue record for this book is available from the Library of Congress.

ISBN 978-1-4020-6086-1 (HB)

ISBN 978-1-4020-6087-8 (e-book)

Published by Springer,

P.O. Box 17, 3300 AA Dordrecht, The Netherlands.

www.springer.com

Printed on acid-free paper

All Rights Reserved

(C) 2007 Springer

No part of this work may be reproduced, stored in a retrieval system, or transmitted in any form or by any means, electronic, mechanical, photocopying, microfilming, recording or otherwise, without written permission from the Publisher, with the exception of any material supplied specifically for the purpose of being entered and executed on a computer system, for exclusive use by the purchaser of the work. 


\section{TABLE OF CONTENTS}

List of Contributors vii

Introduction $\quad x$

PART 1 General Principles of Tumor Immunology

Chapter 1. A Perspective on Cancer Immunology and Immunotherapy Alan N. Houghton

Chapter 2. Tumor Antigens

Michael A. Morse, Timothy M. Clay, and H. Kim Lyerly

Chapter 3. T Cells and Antigen Recognition

Leisha A. Emens

Chapter 4. The Role of Natural Killer T Cells in Tumor Immunity Madhav V. Dhodapkar

Chapter 5. Immunomodulatory Molecules of the Immune System Yvonne M. Saenger, Robert R. Jenq, and Miguel-Angel Perales

Chapter 6. HLA Class I Antigen Abnormalities in Tumors

Barbara Seliger and Soldano Ferrone

Chapter 7. The Local Tumor Microenvironment

Theresa L. Whiteside

\section{PART 2 Tumor Vaccines}

Chapter 8. Peptide and Protein Vaccines for Cancer Raymond M. Wong and Jeffrey S. Weber

Chapter 9. DNA Vaccines Against Cancer Adam D. Cohen and Jedd D. Wolchok 
Chapter 10. Recombinant Viral and Bacterial Vaccines

Douglas W. Grosenbach, Jarett Feldman, Jeffrey Schlom, and Scott I. Abrams

Chapter 11. Dendritic Cell Vaccines

Sylvia Adams, Nina Bhardwaj, and David W. O'Neill

Mark B. Faries and Donald L. Morton

Chapter 13. Carbohydrate Vaccines Against Cancer

Philip O. Livingston and Govind Ragupathi

\section{PART 3 Passive Immunotherapy Approaches}

Chapter 14. Monoclonal Antibody Therapy of Cancer Joseph G. Jurcic, Deborah A. Mulford, and David A. Scheinberg

Chapter 15. Adoptive Cellular Therapy for the Treatment of Cancer Cassian Yee

Chapter 16. Checkpoint Blockade and Combinatorial Immunotherapies 363 Karl S. Peggs, Sergio A. Quezada, and James P. Allison

\section{PART 4 Clinical Tumor Immunotherapy}

Chapter 17. General Approaches to Measuring Immune Responses Mary L. Disis and Keith L. Knutson

Chapter 18. Interferon Therapy

Stergios J. Moschos and John M. Kirkwood

Chapter 19. High Dose Interleukin-2 Therapy

Christian A. Petrulio, Gail DeRaffele, and Howard L. Kaufman

Chapter 20. Monoclonal Antibodies in Cancer Therapy

Christoph Rader and Michael R. Bishop

Chapter 21. Unmasking Tumor Cell Immunogenicity by Chemotherapy: Implications for Therapy

Irma Larma, Robbert G. van der Most, and Richard A. Lake

Index 


\section{LIST OF CONTRIBUTORS}

Scott I. Abrams Laboratory of Tumor Immunology and Biology Center for Cancer Research, National Cancer Institute National Institutes of Health, Bethesda, MD

Sylvia Adams NYU Cancer Institute Tumor Vaccine Center, New York University School of Medicine New York, NY

James P. Allison Howard Hughes Medical Institute, Department of Immunology, Memorial Sloan-Kettering Cancer Center, New York, NY

Nina Bhardwaj NYU Cancer Institute, Tumor Vaccine Center, New York University School of Medicine, New York, NY

Michael R. Bishop Experimental Transplantation and Immunology Branch, Center for Cancer Research, National Cancer Institute, National Institutes of Health, Bethesda, MD

Timothy M. Clay Duke University Medical Center, Durham, NC

Adam D. Cohen Department of Medicine, Memorial Sloan-Kettering Cancer Center, Weill Medical College of Cornell University, New York, NY

Gail DeRaffele The Tumor Immunology Laboratory, Division of Surgical Oncology, Columbia University, New York, NY

Madhav V. Dhodapkar Laboratory of Tumor Immunology and Immunotherapy, The Rockefeller University and Hematology Service, Memorial Sloan Kettering Cancer Center, New York, NY

Mary L. Disis Center for Translational Medicine in Women's Health, Tumor Vaccine Group, University of Washington Seattle, WA

Leisha A. Emens Department of Oncology, Johns Hopkins University School of Medicine, Sidney Kimmel Comprehensive Cancer Center, Baltimore, MD

Mark B. Faries Sonya Valley Ghidossi Vaccine Laboratory of the Roy E. Coats Research Laboratories of the John Wayne Cancer Institute at Saint John's Health Center, Santa Monica, CA 
Jarett Feldman Laboratory of Tumor Immunology and Biology, Center for Cancer Research, National Cancer Institute, National Institutes of Health Bethesda, MD

Soldano Ferrone Hillman Cancer Research Institute, University of Pittsburgh, Pittsburgh, PA

Douglas W. Grosenbach Laboratory of Tumor Immunology and Biology, Center for Cancer Research, National Cancer Institute, National Institutes of Health, Bethesda, MD

Alan N. Houghton Swim Across America Laboratory, Memorial Sloan-Kettering Cancer Center, New York, NY

Robert R. Jenq Memorial Sloan-Kettering Cancer Center, Weill Medical School and Graduate School of Cornell University, New York, NY

Joseph G. Jurcic Memorial Sloan-Kettering Cancer Center, Weill Medical School and Graduate School of Cornell University, New York, NY

Howard L. Kaufman The Tumor Immunology Laboratory, Division of Surgical Oncology, Columbia University, New York, NY

John M. Kirkwood Departments of Medicine and Dermatology, University of Pittsburgh School of Medicine, Melanoma and Skin Cancer Program, University of Pittsburgh Cancer Institute, Pittsburgh, PA

Keith L. Knutson Department of Immunology, Mayo Clinic, Rochester, MN

Richard A. Lake National Research Centre for Asbestos-related Diseases, UWA, Department of Medicine, Sir Charles Gairdner Hospital Perth, Western Australia

Irma Larma National Research Centre for Asbestos-related Diseases, UWA, Department of Medicine, Sir Charles Gairdner Hospital Perth, Western Australia

Philip O. Livingston Laboratory of Tumor Vaccinology, Department of Medicine, Memorial Sloan-Kettering Cancer Center, New York, NY

H. Kim Lyerly Duke Comprehensive Cancer Center, Durham, NC

Michael A. Morse Duke University Medical Center, Durham, NC

Donald L. Morton Sonya Valley Ghidossi Vaccine Laboratory of the Roy E. Coats Research Laboratories of the John Wayne Cancer Institute Melanoma Program at Saint John's Health Center, Santa Monica, CA 
Stergios J. Moschos The University of Pittsburgh Cancer Institute, Melanoma Center, Hillman Cancer Center, Pittsburgh, PA

Deborah A. Mulford Memorial Sloan-Kettering Cancer Center, Weill Medical School and Graduate School of Cornell University, New York, NY

David W. O’Neill NYU Cancer Institute, Tumor Vaccine Center, New York University School of Medicine, New York, NY

Karl S. Peggs Howard Hughes Medical Institute Department of Immunology, Memorial Sloan-Kettering Cancer Center, New York, NY

Miguel-Angel Perales Department of Medicine Memorial Sloan-Kettering Cancer Center, Weill Medical School and Graduate School of Cornell University, New York, NY

Christian A. Petrulio The Tumor Immunology Laboratory, Division of Surgical Oncology, Columbia University, New York, NY

Sergio A. Quezada Howard Hughes Medical Institute, Department of Immunology Memorial Sloan-Kettering Cancer Center, New York, NY

Christoph Rader Experimental Transplantation and Immunology Branch, Center for Cancer Research, National Cancer Institute, National Institutes of Health Bethesda, MD

Govind Ragupathi Laboratory of Tumor Vaccinology, Department of Medicine Memorial Sloan-Kettering Cancer Center, New York, NY

Yvonne M. Saenger Memorial Sloan-Kettering Cancer Center, Weill Medical School and Graduate School of Cornell University, New York, NY

David A. Scheinberg Memorial Sloan-Kettering Cancer Center, Weill Medical School and Graduate School of Cornell University, New York, NY

Jeffrey Schlom Laboratory of Tumor Immunology and Biology, Center for Cancer Research, National Cancer Institute, National Institutes of Health, Bethesda, MD

Barbara Seliger Institute of Medical Immunology, Martin Luther University Halle, Germany

Robbert G. van der Most National Research Centre for Asbestos-related Diseases, UWA, Department of Medicine, Sir Charles Gairdner Hospital Perth, Western Australia 
Jeffrey S. Weber Donald A. Adam Comprehensive Melanoma Research Center, H. Lee Moffitt Cancer Center and Research Institute, Tampa, FL

Theresa L. Whiteside University of Pittsburgh Cancer Institute, Departments of Pathology, Immunology and Otolaryngology, University of Pittsburgh School of Medicine, Pittsburgh, PA

Jedd D. Wolchok Department of Medicine, Memorial Sloan-Kettering Cancer Center, Weill Medical College of Cornell University, New York, NY

Raymond M. Wong Department of Molecular Microbiology and Immunology and Medicine, Keck School of Medicine, University of Southern California, Los Angeles, CA

Cassian Yee Program in Immunology, Clinical Research Division, Fred Hutchinson Cancer Research Center, University of Washington, Seattle, WA 


\section{INTRODUCTION}

As stated so elegantly by Dr. Alan Houghton in Chapter 1 of General Principles of Tumor Immunotherapy: Basic and Clinical Applications of Tumor Immunology, the connection between infection and tumor immunity has been recognized for millennia. However, it is only in the last few decades that the molecular and cellular basis for tumor immunotherapy been elucidated. The promise for manipulating the immune system to fight cancer is enormous given the fine specificity of immune responses and the ability to develop memory responses allowing long term protection from recurrent disease. The practical application of tumor immunotherapy, however, has lagged behind the promise. This is partly due to our incomplete understanding of how the host immune system interacts with tumor cells and partly due to the slow nature of clinical and translational research. Nonetheless, a clearer understanding of the complex host-tumor interactions coupled with new insight from two decades of productive clinical trial activity provides new enthusiasm for the use of tumor immunotherapy in the armamentarium of therapeutic strategies for patients with cancer.

General Principles of Tumor Immunotherapy: Basic and Clinical Applications of Tumor Immunology seeks to bring together the most current information related to how the immune system recognizes and eradicates cancer with a particular focus on the application of tumor immunotherapy in the clinic. This volume is organized into four sections designed to focus on particular aspects of tumor immunotherapy. In Part I the basic principles upon which tumor recognition and rejection are based will be discussed and includes chapters on the identification of tumor antigens, the mechanisms used to present tumor antigens to the immune system, features of the innate and adaptive immune systems as they relate to tumor immunology and an examination of the tumor microenvironment as it relates to host-tumor interactions. In Part II a highly focused discussion of the various active vaccine strategies that have been brought forward over the last decade is presented. In Part III we focus on the role of passive immunotherapy in cancer treatment. Finally, in Part IV we discuss some of the current clinical applications of immunotherapy and provide a provocative discussion on the future of combination therapy utilizing immunotherapy and more standard cancer therapeutics. These chapters have been authored by world class tumor immunologists and clinical investigators dedicated to pursuing the potential of tumor immunotherapy.

There are many people to thank when writing a book such as this. First, we want to thank all of our authors who so willingly agreed to contribute to this endeavor. The final product speaks for itself. We also want to express our sincerest gratitude to Dr. Alan Houghton, who not only provided one of the most comprehensive 
and insightful reviews of the history of tumor immunotherapy, but also provided significant inspiration in the pursuit of writing this book. Finally we want to thank Melania Ruiz and the folks at Springer for their encouragement, patience, and dedication to excellence. Our hope is that this volume will be a useful guide to those scientists and physicians who seek to understand the current status of tumor immunotherapy and the basic biology that supports its use as a cancer therapeutic. We also hope that this book will help motivate the students of tumor immunology and immunotherapy to keep working in this important and exciting field.

Howard L. Kaufman, MD

Jedd D. Wolchok, MD, PhD 